
allemande

51-1 | 2019

La République démocratique allemande dans l'espace public européen (1949-2018)

\title{
Le 17 juin 1953 dans la presse ouest-allemande et française
}

Nadine Willmann

\section{(2) OpenEdition \\ Journals}

Édition électronique

URL : https://journals.openedition.org/allemagne/1561

DOI : $10.4000 / a l l e m a g n e .1561$

ISSN : 2605-7913

Éditeur

Société d'études allemandes

Édition imprimée

Date de publication : 2 juillet 2019

Pagination : 113-128

ISSN : 0035-0974

Référence électronique

Nadine Willmann, «Le 17 juin 1953 dans la presse ouest-allemande et française », Revue d'Allemagne et des pays de langue allemande [En ligne], 51-1 | 2019, mis en ligne le 02 juillet 2020, consulté le 01 juin 2022. URL : http://journals.openedition.org/allemagne/1561 ; DOI : https://doi.org/10.4000/

allemagne. 1561 


\section{Le 17 juin 1953 dans la presse ouest-allemande et française}

\section{- Nadine Willmann*}

Lors de la deuxième conférence du SED de juillet 1952, Walter Ulbricht, secrétaire général du SED et vice-ministre-président de la RDA, proclama la construction accélérée du socialisme. Les entreprises privées devaient se raréfier et la production industrielle lourde se développer aux dépens de la production de biens de consommation, entre autres mesures ${ }^{(1)}$. La fin de l'année fut émaillée de grèves, et l'exode vers l'Ouest s'accrut $^{(2)}$. Le 14 mai 1953, la 13e session du comité central du SED annonça une augmentation des normes de travail de $10 \%$ au $1^{\text {er }}$ juin ${ }^{(3)}$, ce qui se traduisait par une perte de salaire de 20 à $30 \%{ }^{(4)}$. Début juin, Ulbricht et Otto Grotewohl, le ministreprésident, furent convoqués à Moscou, où Nikita Khrouchtchev leur signifia de relayer la politique menée les derniers mois par un "nouveau cours", qui devait revenir sur ces évolutions ${ }^{(5)}$. Le 9 juin, le bureau politique du SED publia un communiqué entérinant ces décisions, qui fut perçu favorablement par la population, mais aussi interprété comme un aveu de faiblesse du régime. En outre, beaucoup furent déçus du maintien de l'élévation des normes de production ${ }^{(6)}$. Ce sont deux facteurs déclencheurs du soulèvement du 17 juin.

Ce bref rappel du contexte était nécessaire pour aborder notre propos, la représentation de l'insurrection, qui suscita dans l'espace public occidental un intérêt considérable, autant dans la Frankfurter Allgemeine Zeitung, la Süddeutsche Zeitung, la Frankfurter Rundschau et Die Welt, journaux ouest-allemands, que dans Le Figaro,

\footnotetext{
* Professeur agrégé d'allemand et docteur en études germaniques, Institut d'études politiques de Strasbourg.

1 Thomas Flemming, Kein Tag der deutschen Einheit - 17. Juni 1953, Berlin, be.bra.Verlag, 2003, p. 17-18.

2 Ibid., p. 21.

3 Ilko-Sascha Kowalczuk, 17. Juni 1953, Munich, Beck, 2013, p. 21-22.

4 T. Flemming, Kein Tag der deutschen Einheit (note 1), p. 36.

5 Ibid., p. 36-37.

6 I. Kowalczuk, 17. Juni 1953 (note 3), p. 29.
} 
Le Monde, La Croix et L'Humanité. Notre objectif consiste à comparer la perception des presses ouest-allemande et française, afin, d'une part, de mettre éventuellement en lumière une vision nationale des faits, et d'autre part, de faire ressortir les différences entre journaux conservateurs, comme la FAZ, Die Welt, Le Figaro et La Croix, et journaux progressistes, comme la Süddeutsche Zeitung, la Frankfurter Rundschau, Le Monde et L'Humanité. On est en effet en droit de s'interroger sur l'existence éventuelle d'une pensée conservatrice et d'une pensée progressiste transnationales. Nous avons étudié les articles publiés entre le 17 juin et le 16 juillet - étant entendu qu'ils rapportent toujours les faits de la veille. Nous envisagerons successivement différentes facettes des événements: l'insurrection proprement dite, le gouvernement, la répression, le rôle des Soviétiques et le thème de la réunification, et, enfin, les articles de fond.

\section{Une représentation de l'insurrection et des interprétations similaires dans tous les journaux}

Le 17 juin, tous les journaux rendent compte des manifestations massives nées sur la Stalinallee, le chantier de prestige de Berlin-Est, et aboutissant à la Maison des ministères. Les manifestants réclament notamment le retrait de l'augmentation des normes de production, la démission du gouvernement et des élections libres. La Süddeutsche Zeitung rapporte de manière plus colorée comment un ouvrier torse nu, grimpé sur une table, proclame: «Nous voulons être libres!» ${ }^{(7)}$. Die Welt est d'emblée plus sensationnaliste: «Des milliers d'ouvriers ont imposé leur volonté au gouvernement de Pankow», qui «capitula» en annulant la décision relative aux normes de production ${ }^{(8)}$. La Frankfurter Rundschau prend du recul: cette première protestation spontanée de masse contre le régime est une «tornade politique» aux conséquences imprévisibles ${ }^{(9)}$. L'Humanité réduit les événements à un cortège paisible d'ouvriers réclamant le maintien des normes de production et résistant aux tentatives de "provocateurs » pour faire dégénérer la manifestation ${ }^{(10)}$.

Le lendemain, tous les quotidiens font état de la grève générale, de nouvelles manifestations et de l'activation de l'état de siège. La FAZ témoigne de la violence de certains manifestants, qui pillent et incendient des magasins ${ }^{(11)}$. Plusieurs quotidiens signalent que deux jeunes gens grimpent sur la porte de Brandebourg et tentent de remplacer le drapeau rouge par un drapeau noir-rouge-or ${ }^{(12)}$. Encore une fois, la Süddeutsche Zeitung s'avère plus anecdotique que la $F A Z$, citant des exclamations crues de manifestants à l'adresse d'apparatchiks: «Ferme-la, tu ne fais que vivre de notre argent! » ${ }^{(13)}$. Die Welt

7 «Demonstrationen in Ostberlin», Süddeutsche Zeitung (par la suite SZ), 17.06.1953.

8 «Erste Rebellion gegen SED-Regime», Die Welt (par la suite W), 17.06.1953.

9 «Arbeiter demonstrieren gegen das SED-Regime», Frankfurter Rundschau (par la suite FR), 17.06.1953.

10 Rosa Michel, «Le Bureau Politique du Parti Socialiste Unifié d’Allemagne condamne l'application bureaucratique des décisions sur les normes de production ", L'Humanité (par la suite H), citée d'après Ulrich Pfeil, "Comme un coup de tonnerre dans un ciel d'été» - Französische Reaktionen auf den 17. Juni 1953, Berlin, Berliner Wissenschafts-Verlag, 2003, p. 50.

11 «Der Ausnahmezustand über Berlin verhängt», FAZ, 18.06.1953.

12 «Die Rote Fahne niedergeholt», FAZ, 18.06.1953. Aussi dans le $S Z$ et la FR. Les trois couleurs symbolisent unité, liberté et démocratie depuis le XIX ${ }^{e}$ siècle, mais n'ont officiellement représenté la nation allemande que depuis la République de Weimar.

13 «'Wir haben lange genug rot gesehen'», SZ, 18.06.1953. 
remarque que grèves et manifestations ont atteint la province ${ }^{(14)}$. Le Figaro mentionne le nombre important de Vopos (Volkspolizisten) s'étant placés sous la protection de la police occidentale pour éviter de participer à la répression ${ }^{(15)}$. Le Monde livre un tableau coloré de la Potsdamer Platz:

«De tous côtés brûlent les panneaux de propagande et les kiosques à journaux, tandis que les chars russes [...] se déploient [...]. Des pluies de pierres ricochent sur leur blindage au milieu des cris et des huées » ${ }^{(16)}$.

L'Humanité, seule voix discordante dans le concert des journaux étudiés, reprend à son compte la thèse du gouvernement de RDA et affirme que des "provocateurs fascistes et autres réactionnaires à la solde de puissances étrangères et des monopoles capitalistes allemands ont provoqué de graves désordres à Berlin-Est», exploitant le mécontentement des ouvriers «face à l'application bureaucratique de l'augmentation des normes de production ${ }^{(17)}$ et faisant débrayer les ouvriers dans les usines à force de menaces, mais se heurtant à la population, qui put disperser «les bandits » avec l'aide de la police ${ }^{(18)}$.

Le 19 juin, la Süddeutsche Zeitung ne dédaigne pas le ton mélodramatique et déclamatoire:

«En l'espace de 36 heures s'est joué un drame à Berlin-Est qui, à travers un voile de sang et de nuages de fumée, a laissé entrevoir [...] ce qui se dissimule sinon sous la surface d’une tyrannie [...]. Cet instant a suffi à suspendre la respiration du monde ${ }^{(19)}$.

L'Humanité assure que le gouvernement est-allemand crée les prémices pour de véritables élections libres dans toute l'Allemagne et qu'Adenauer redoute cette perspective, d'où l'action des «agents occidentaux» le 17 juin $^{(20)}$. Le quotidien affirme ainsi que des officiers américains se trouvaient parmi les manifestants "pour diriger leurs agents fascistes » et que des avions américains survolaient Berlin-Est en lançant des tracts. En outre, le journaliste prétend que la population a pris fait et cause pour les autorités ${ }^{(21)}$. Enfin, il ne peut passer sous silence l'intervention des chars soviétiques, tout en s'en félicitant: «Seule la puissance d'occupation a défendu la ville avec la fermeté qui s'imposait » ${ }^{(22)}$.

Les 21-22 juin, Die Welt rapporte que le SED a enfin reconnu que les émeutes touchent l'ensemble du territoire ${ }^{(23)}$ et confirme que la ligne de démarcation entre l'Est et l'Ouest de Berlin est toujours fermée ${ }^{(24)}$. Le journal prise encore une fois le sensationnalisme:

14 «Ausstrahlung auf die Ostzone», W, 18.06.1953. Aussi dans la FR.

15 «Grèves et troubles dans plusieurs villes », Le Figaro (par la suite F), 18.06.1953.

16 Roland Delcour, «Les chars soviétiques ouvrent le feu... », Le Monde (par la suite M), 18.06.1953.

17 Rosa Michel, «À Berlin, graves provocations des revanchards», H, 18.06.1953.

18 Rosa Michel, «Les provocations revanchardes», H, 18.06.1953.

19 Werner FriedmanN, «Signal für Ost und West», SZ, 19.06.1953.

20 «À Berlin, des officiers américains dirigeaient les groupes de provocateurs, raconte le 'Neues Deutschland'», $H, 19.06 .1953$.

21 Ibid.

22 Ibid.

23 «SED gibt Aufstand in der Ostzone zu», W, 20-21.06.1953.

24 «Verhaftungen in Ost-Berlin», W, 20-21.06.1953. 
«Avec chaque pas frappé sur le pavé par les soldats de l'Armée rouge et les policiers avec leurs chaussures cloutées, s'effrite la résistance d'une communauté d'ouvriers qui s’apprêtait, comme collectif, à tordre le cou à leur gouvernement qui leur a inculqué le collectif» ${ }^{(25)}$.

La Süddeutsche Zeitung fait état des troubles observés dans toute la RDA, avec retard par rapport aux autres journaux, néanmoins avec plus de détails ${ }^{(26)}$. Le Monde est le premier à témoigner de révoltes dans le monde rural, «où les paysans auraient attaqué à coups de fourche la police et les fonctionnaires du parti » ${ }^{(27)}$. L'Humanité pour sa part affirme que Berlin-Est s'est remis au travail et que «de tout le pays, de toutes les couches de la population, affluent au Comité Central du Parti Socialiste Unifié des manifestations de fidélité et de confiance» ${ }^{(28)}$. Le 22 juin, la Süddeutsche Zeitung, à l'image d'autres journaux, signale que le calme est peu à peu revenu à Berlin-Est. Le Figaro sacrifie au pathos:

«[...] à l'heure où nous avons constaté que le soulèvement d'un tronçon de peuple, affamé et tenu sous la botte par des vainqueurs sans merci et leurs hommes de main, constitue dans l'histoire du totalitarisme de l'Est un acte de courage sans précédent » ${ }^{(29)}$.

L'Humanité transmet le témoignage d'un «agent provocateur» de l'Ouest, payé pour fomenter des troubles et commettre des dégradations à Berlin-Est, sur instructions d'un homme travaillant pour la police de Berlin-Ouest ${ }^{(30)}$. Le 23 juin, le journal expose comme un fait établi ce que ses confrères mettent en doute: l'épisode d'avions étrangers amenant en RDA des "groupes aéroportés de bandits pourvus d'armes et d'émetteurs» et de camions remplis d'armes sur l'autoroute ${ }^{(31)}$. En outre, le quotidien, citant une déclaration du comité central du SED, étoffe son accusation contre l'Ouest:

«Plaçant les événements du 17 juin dans le cadre de la situation internationale, la déclaration montre que le 17 juin a été choisi comme 'jour J' d'une tentative pour rallumer, en partant de Berlin pour le déployer en République démocratique allemande, un foyer de guerre ${ }^{(32)}$.

Le 24 juin, la Süddeutsche Zeitung publie d'ailleurs un communiqué du SED sur l'insurrection exposant la thèse du «jour J» envisagé depuis longtemps par une agence gouvernementale de RFA pour déclencher un soulèvement en RDA afin de contrecarrer le changement de cap visant une amélioration des conditions de vie; le journaliste stigmatise «la langue - qui nous est connue depuis l'époque nazie - d'un régime pressentant son déclin, laquelle mêle mots triomphants et concessions penaudes » ${ }^{(33)}$. Un correspondant du Monde revient sur le 16 juin, où, sur la Stalinallee, il a observé les attroupements d'ouvriers harangués par des orateurs improvisés, et il dément la thèse des provocateurs occidentaux, avant de tirer deux conclusions: d'une part, le rideau

25 Wolfgang WeINERT, «Schweigen hinter roten Fahnen», W, 20-21.06.1953.

26 "Streiks und Demonstrationen in der ganzen Sowjetzone», SZ, 20-21.06.1953.

27 Roland Delcour, «'L’ordre règne à Berlin-Est'», $M, 20-21.06 .1953$.

28 Rosa Michel, «La réponse du peuple allemand», H, 20-21.06.1953.

29 Dominique Auclères, "Amorce de détente à Berlin-Est», F, 22.06.1953.

30 Rosa Michel, «À Berlin un agent américain révèle comment l'émeute fasciste a été fomentée», $H$, 22.06.1953.

31 Rosa Michel, «Le parti socialiste unifié d'Allemagne prend de nouvelles décisions pour améliorer le niveau de vie de la population », $H, 23.06 .1953$.

32 Ibid.

33 «Ein Dokument der Verwirrung», SZ, 24.06.1953. 
de fer est vital au régime - on ne laisse pas impunément l'ouvrier de la zone soviétique circuler librement dans l'univers capitaliste; d'autre part, les scènes de rues de ces journées laissent entrevoir ce qui se passerait si les troupes soviétiques évacuaient Berlin-Est: un «immense règlement de compte avec une brutalité inouïe " ${ }^{(34)}$. L'Humanité évoque le cas d'Erna Dorn, retraçant le parcours de cette ancienne commandante de camp de concentration qui aurait «dirigé les émeutiers fascistes " ${ }^{(35)}$, mais concède que les provocateurs n'auraient pu susciter de troubles si graves s'ils n'avaient trouvé un terrain favorable. Et le journal d'évoquer des «nostalgiques» de l'hitlérisme en Allemagne, combattus à l'Est mais encouragés à l'Ouest, reprenant ici aussi l'analyse des événements par la presse de $\mathrm{RDA}^{(36)}$.

Le 25 juin, L’Humanité poursuit son réquisitoire contre «les coalisés atlantiques » qui font de Berlin-Est «leur base de guerre» et réaffirme que le mécontentement des ouvriers n'a été que «l'occasion exploitée par des bandes organisées pour essayer de camoufler en révolte ouvrière une tentative de putsch " ${ }^{(37)}$. La Süddeutsche Zeitung rappelle le 26 que le calme n'est pas encore totalement revenu et que de nombreuses grèves ont encore lieu dans les usines ${ }^{(38)}$. Die Welt chiffre à 146 le nombre de policiers est-allemands ayant fui à Berlin-Ouest depuis les événements ${ }^{(39)}$. Le 27, Le Figaro rapporte que des milliers de Berlinois ont manifesté sous une pluie battante en faveur du régime communiste, ce que ne manque pas de souligner également L'Humanitét ${ }^{(40)}$. Cette dernière retrace la genèse en RFA du plan concernant «le jour J, en vue de l'annexion de la zone soviétique», dit en Allemagne Tag $X$, plan concocté lors d'une conférence à Berlin-Ouest entre représentants de trusts d'armement et du gouvernement, "ainsi que les chefs de différentes organisations fascistes». On aurait mis sur pied un état-major auprès du ministre fédéral pour les Questions allemandes (Bundesminister für Gesamtdeutsche Fragen), à savoir Jakob Kaiser, dont la tâche principale résidait dans l'organisation et le financement d'un réseau d'agents en $\mathrm{RDA}^{(41)}$. Le 30, le même quotidien publie les aveux d'un meneur d'émeutiers, ancien membre des SA ${ }^{(42)}$. Le 7 juillet, la FAZ mentionne les grèves et actions de travail au ralenti menées par les ouvriers pour obtenir la libération de leurs collègues emprisonnés ${ }^{(43)}$, tandis que L'Humanité se félicite des manifestations de solidarité envers le gouvernement et de protestation contre «les provocations fascistes " ${ }^{(4)}$. Le 9, la Süddeutsche Zeitung signale également des grèves sur le tas pour la libération des grévistes du 17 juin. Les ouvriers attendent du ministre de la Justice Max Fechner, qui avait stipulé que le droit de grève était garanti par la constitution, qu'il

César SAntelli, "Que signifient les émeutes de Berlin-Est?», M, 24.06.1953.

«L'ex-commandante SS de Ravensbruck dirigeait à Halle les émeutiers fascistes», H, 24.06.1953.

«Le drapeau rouge de Berlin», H, 24.06.1953.

«La vérité sur les événements », H, 25.06.1953.

«Acht Tage nach dem Berliner Aufstand», SZ, 26.06.1953.

«46 an einem Tag», W, 26.06.1953.

"Manifestations pro-gouvernementales à Berlin-Est», F, 27-28.06.1953 et «Puissante manifestation à Berlin-Est contre les provocateurs fascistes», H, 27-28.06.1953.

"Qui était derrière les émeutiers fascistes de Berlin?», H, 27.06.1953.

«L'un des meneurs émeutiers était un chef des SA», H, 30.06.1953.

«Noch Streiks in der Sowjetzone», FAZ, 07.07.1953.

Rosa Michel, «Puissantes manifestations en Allemagne démocratique contre les provocateurs fascistes et en faveur des mesures prises par le gouvernement», H, 07.07.1953. 
agisse en conséquence ${ }^{(45)}$. Le 13, La Croix révèle que 752 réfugiés est-allemands se sont présentés à Berlin-Ouest en une seule journée ${ }^{(46)}$.

On constate à l'issue de ce premier chapitre sur l'insurrection que la peinture des événements ne diffère guère selon qu'elle émane de journaux français ou ouest-allemands, ou bien de la presse conservatrice ou progressiste, on voit par conséquent émerger dans ces articles une pensée conservatrice et une pensée progressiste transnationales. Seule L'Humanité figure une voix discordante dans ce chorus, elle qui reprend à son compte la thèse du gouvernement d'une tentative de putsch occidental. Ce qui semble le plus ici distinguer les journaux, c'est - hormis leur choix de rapporter différents faits - le ton qu'ils adoptent, sobre dans la FAZ, imagée et anecdotique dans la Süddeutsche Zeitung, sensationnaliste dans Die Welt, voire empreint de pathos dans Le Figaro.

\section{Un regard singulièrement sévère de la presse française sur le régime}

Le 17 juin, Le Monde, citant le Telegraf sous licence britannique, prétend que Grotewohl aurait affirmé que seule la démission d'Ulbricht, secrétaire général du SED et vice-ministre-président de la RDA, pouvait sauver le gouvernement ${ }^{(47)}$. Le lendemain, la Frankfurter Rundschau affirme que dans les entreprises, des apparatchiks du SED font cause commune avec les insurgés ${ }^{(48)}$, avant de dépeindre une réunion de responsables du parti devant laquelle Grotewohl et Ulbricht promettent des mesures pour élever le niveau de vie ${ }^{(49)}$. Le lendemain, Le Figaro tire de ce «véritable mouvement révolutionnaire» des conclusions aux allures de guerre froide: «La journée du 17 juin [...] a montré ce qu'il faut penser du 'paradis' ouvert aux masses ouvrières par le régime communiste et détruit un mythe qu'on ne reconstruira pas aisément ${ }^{(50)}$. On décèle ici une inclination de la presse française à mettre en lumière, plus que la presse ouest-allemande, l'impéritie du régime. Le Monde impute le soulèvement au «nouveau cours", qui revenait sur l'annonce d'une politique de socialisme accéléré, ajoutant qu'«un mécontentement ouvrier généralisé couve depuis plusieurs mois dans toute la zone orientale», avant de conclure, enclin ici aussi à réprouver durement le régime: «Le danger est mortel pour un régime communiste en pleine construction que de se voir ainsi abandonné par le prolétariat dont il prétend instaurer la dictature» ${ }^{(51)}$.

Le 19 juin, de nouveau, Le Figaro se livre à une apologie du peuple soulevé et à une attaque en règle du gouvernement: «Le peuple opprimé et exploité a été convaincu que le gouvernement soviétique se désintéressait de la clique des dirigeants allemands mise en place par lui. Il pensa se libérer de ses bourreaux» ${ }^{(52)}$. Même attaque dans Le Monde, qui dit le gouvernement «souillé du sang de ces travailleurs dont il se prétend le représentant» ${ }^{(53)}$. La presse française non communiste semble se plaire plus que sa

45 «Schlagbäume in Berlin gehen hoch», SZ, 09.07.1953.

46 «À Berlin et en Allemagne orientale», La Croix (par la suite C), 12-13.07.1953.

47 «Troubles graves à Berlin-Est», $M, 17.06 .1953$.

48 «Ausnahmezustand in Ost-Berlin», FR, 18.06.1953.

49 «Ulbricht und Grotewohl: Wir irrten», FR, 18.06.1953.

50 Roger Massıp, «Véritable révolte anticommuniste hier à Berlin-Est», F, 18.06.1953.

51 «Une révolte ouvrière», $M, 18.06 .1953$.

52 Dominique Auclères, “'J'ai vu les troupes soviétiques reprendre en main la situation' », F, 19.06.1953.

53

Roland Delcour, «Les autorités de Berlin-Est ont perdu le bénéfice des mesures de détente», M, 19.06.1953. 
consœur ouest-allemande à souligner les méfaits du régime et l'erreur qui consisterait à considérer le monde communiste comme une zone de bonheur pour les citoyens. Le 20-21 juin, la Frankfurter Rundschau détecte l'apparition au sein du SED d'un «mouvement titiste» qui impute à Ulbricht la responsabilité des événements ${ }^{(54)}$. Le Figaro enregistre avec ironie un «chapelet d'autocritiques » de la part des dirigeants, "paroles sibyllines qui peuvent préluder au crépuscule de ces faux dieux plus vraisemblablement qu'au durcissement de leur régime» ${ }^{(55)}$.

Le 22 juin, Die Welt se fait l'écho de bruits sur un remaniement du gouvernement, à l'initiative du haut-commissaire soviétique Semionov, avec un remplacement d'Ulbricht et une plus forte participation des partis bourgeois (ces partis dits "partis de bloc» tolérés qui ne représentent pas réellement une opposition) ${ }^{(56)}$. Le Figaro envisage également l'attribution à Otto Nutschke, leader de la CDU chrétienne-démocrate, un des partis bourgeois de RDA, de responsabilités plus étendues au sein du pouvoir, qui empiéteraient sur celle du SED ${ }^{(57)}$, instaurant ainsi une sorte de pluralisme politique. Le 23, à l'instar d'autres publications, la $F A Z$ résume un programme de neuf points présenté par le comité central du SED, concernant le renforcement de l'appareil du parti, la limitation de l'industrie lourde au bénéfice des biens de consommation et le retour aux normes de production antérieures. Le journal cite le communiqué où le SED reconnaît que, quand les masses ne comprennent plus le parti, c'est le parti qui en porte la faute, et non les ouvriers, et qu'il importe donc de se rapprocher de ces derniers ${ }^{(58)}$. La Süddeutsche Zeitung décrit par ailleurs le développement par le SED de la thèse d'agents instigateurs occidentaux, qui auraient organisé une croisière en bateau à vapeur sur le Müggelsee pour le personnel d'une entreprise, dans laquelle aurait ensuite été fomentée la rébellion ${ }^{(59)}$. Le 24 , Die Welt analyse l'action du gouvernement comme une combinaison de sanctions contre les manifestants et d'efforts pour regagner la confiance des ouvriers $^{(60)}$, qui ne parviennent pas à empêcher d'autres manifestations pour la liberté et contre la pénurie d'aliments ${ }^{(61)}$. Le Figaro indique le 24 que Grotewohl reconnaît ses erreurs et, tout en refusant de démissionner, s'engage à en tirer les conséquences ${ }^{(62)}$.

Le 25 juin, comme les autres journaux, la $F A Z$ rend compte des premières visites de Ulbricht et Grotewohl dans les entreprises, où, devant le personnel, ils reconnaissent de grandes fautes qu'aurait commis le parti et exposent le «Nouveau cours» ${ }^{(63)}$. De leur côté, la Süddeutsche Zeitung et la Frankfurter Rundschau, attestant ici une

54 «Sowjets noch nicht Herren der Lage», FR, 20-21.06.1953. Le titoisme ou titisme consiste en une recherche par un pays de la voie du socialisme en utilisant ses propres ressources et en usant de politiques adaptées à son propre contexte national, plutôt que de se conformer à une politique dictée de l'extérieur, en l'occurrence par l'URSS.

55 Dominique Auclères, «La répression soviétique s'étend à toute la zone orientale», F, 20-21.06.1953.

56 «Verhaftungen gestoppt?», W, 22.06.1953.

57 D. Auclères, «Amorce de détente à Berlin-Est» (note 29).

58 «Selbstanklagen und neue Zugeständnisse», FAZ, 23.06.1953.

59 «Die SED kämpft verzweifelt um ihre Positionen», SZ, 23.06.1953.

60 «Standgericht in Jena», W, 24.06.1953.

61 «Hungerdemonstrationen in Sachsen und Thüringen», W, 24.06.1953.

62 «M. Grotewohl 'reconnaît ses erreurs'», F, 24.06.1953.

63 «Ulbricht und Grotewohl wollen beruhigen», FAZ, 25.06.1953. 
tradition progressiste commune, déplacent à ce propos l'accent sur les réactions âpres des ouvriers ${ }^{(64)}$ : «J'entends bien le message", dit un ouvrier citant le Faust de Goethe, «mais la foi me manque» ${ }^{(65)}$. À l'inverse, selon L'Humanité, les interventions d'Ulbricht et Grotewohl en usines seraient «de puissantes manifestations de coopération fraternelle entre les travailleurs et leur gouvernement» ${ }^{(66)}$. Le 26, la $F A Z$ signale que le gouvernement a annoncé que tous les programmes militaires ont été annulés au profit de l'approvisionnement civil et que des réserves alimentaires d'État stockées pour la future armée nationale ont été débloquées ${ }^{(67)}$. La Süddeutsche Zeitung indique elle aussi que Grotewohl le premier a pris ses distances avec la thèse du gouvernement sur le soulèvement: "C'est nous qui en portons la responsabilité » et la situation qui a permis ces troubles n'a été «en aucune façon la seule œuvre de provocateurs occidentaux " ${ }^{(68)}$. Selon Le Figaro, le SED «marche d'une humiliation à l'autre», Grotewohl avouant à propos des réfugiés à l'Ouest qu'il s'agit notamment de paysans dont le départ provoque une grave crise de ravitaillement ${ }^{(69)}$.

Le 27 juin, selon la $F A Z$, Ulbricht a déclaré qu'une grève dans l'État des travailleurs est une entaille portée par la classe ouvrière dans sa propre chair, lui déniant ainsi le droit de grève ${ }^{(70)}$. La Frankfurter Rundschau est la première à augurer de grands changements à la direction du SED: Ulbricht serait maintenu, mais le poste de secrétaire général devrait être aboli et un présidium devrait remplacer le Politbüro à la tête du parti. Par ailleurs, le responsable de la propagande, Hermann Axen, déplore que la presse et la radio est-berlinoises enjolivent les faits ${ }^{(71)}$. La Croix se perd en conjectures sur le sort du gouvernement est-allemand: Grotewohl devrait être remplacé à sa tête par Hermann Kastner, ancien président du Liberal-demokratische Partei (LDP), l'un des «partis de bloc» et vice-ministre-président déchu puis réhabilité(72). Le 29, la Süddeutsche Zeitung fait état de départs massifs du parti de membres du SED, pour la raison que ce parti «aurait trahi la cause de la classe des travailleurs » ${ }^{(73)}$. Le Monde reprend le thème de l'«humiliation» du gouvernement, Grotewohl reconnaissant que lui et ses collègues portent la responsabilité de l'état de choses qui a conduit aux émeutes. Le même journaliste, évoquant le nombre réduit d'ouvriers venus écouter Ulbricht dans les entreprises, ajoute que l'épreuve de force entre le gouvernement et la classe ouvrière s'est transportée de la rue dans l'usine ${ }^{(74)}$. La Croix en rajoute dans la même veine, teintée de pathos:

64 «SED-Politiker wollen nicht abtreten», SZ, 25.06.1953.

65 «Grotewohl und Ulbricht wollen nicht abtreten», FR, 25.06.1953.

66 Rosa Michel, «Dans les usines, Grotewohl, Ulbricht, Ebert et Hernstadt prennent la parole devant les travailleurs », H, 25.06.1953.

67 «Die Sowjetzone gibt die Reserven frei», FAZ, 26.06.1953.

68 «Acht Tage nach dem Berliner Aufstand» (note 38).

69 «Le trafic normal entre les deux Berlin serait rétabli demain», F, 26.06.1953.

70 «Vor der Aufhebung des Ausnahmezustands?», FAZ, 27-28.06.1953.

71 «Umbau der SED-Führung angekündigt», FR, 27-28.06.1953.

72 «Le gouvernement Grotewohl prend des mesures pour améliorer le sort des Allemands de l'Est», C, 27-28.06.1953.

73 «Massenaustritte aus der SED», SZ, 29.06.1953.

74 Roland Delcour, «Les dirigeants communistes de Berlin-Est auront fort à faire pour reconquérir la confiance des ouvriers », $M, 29.06 .1953$. 
«Si une chose est certaine dans le drame des jours derniers, c'est - autant que l'admirable courage dont a fait preuve le Berlinois bravant des chars blindés avec la seule arme de ses poings nus - le discrédit total avec lequel le gouvernement de marionnettes d'Allemagne orientale sort de l'aventure» ${ }^{(75)}$.

On vérifie ici combien la presse française est impitoyable dans son réquisitoire. Enfin, La Croix cite le président de la fédération des syndicats est-allemands, Herbert Warnke, fustigeant les "violations des lois démocratiques commises par les autorités » et déplorant que les directions syndicales aient oublié de défendre les intérêts des travailleurs ${ }^{(76)}$.

Le $1^{\text {er }}$ juillet, la $F A Z$, parmi d'autres, mentionne les télégrammes envoyés à Ulbricht pour son $60^{\mathrm{e}}$ anniversaire par les instances soviétiques et le SED, qui attestent que les rumeurs de démission étaient prématurées, même si les rituelles biographies et émissions spéciales font cette fois défaut ${ }^{(77)}$. Toutefois, les 4 et 5 juillet, la FAZ fait état de dissensions, au sein du cabinet ministériel, entre membres du SED et représentants des partis bourgeois, notamment sur le maintien d'Ulbricht comme vice-ministre-président $^{(78)}$. La Frankfurter Rundschau ajoute que la CDU s'efforce d'étendre le «Nouveau cours » à l'agriculture, s'opposant au souhait du SED de maintenir tous les kolkhozes ${ }^{(79)}$. Le 8 juillet, à l'instar d'autres journaux, la Süddeutsche Zeitung déclare que le mécontentement a envahi les rangs du SED et que de nombreux insignes du parti se sont «égarés ", tandis que la CDU et le LPD ont été chargés par les autorités d'établir des listes de personnes aptes à remplir des missions de gestion ${ }^{(80)}$. La Frankfurter Rundschau observe une fuite similaire des effectifs au sein de la fédération syndicale, le FDGB ${ }^{(81)}$. Le 13, le même quotidien fait état de la mise en œuvre du "Nouveau cours» sous différentes formes: libération de prisonniers, activation des partis bourgeois, assurance que l'Église ne serait plus empêchée dans son travail auprès de la jeunesse ${ }^{(82)}$.

Le 14 juillet, la $F A Z$, rapportant que lors de leurs tournées dans les usines, les représentants du gouvernement proposent l'amélioration de la situation sanitaire comme un exutoire pour le mécontentement des ouvriers, persifle: "On tente d'apaiser un soulèvement populaire [...] par le biais de lavabos triples et de réfrigérateurs réparés " ${ }^{(83)}$. La Süddeutsche Zeitung se fait l'écho d'une déclaration du commentateur radiophonique Karl-Eduard von Schnitzler ${ }^{(84)}$ quant au retour de l'humour et de la bonne humeur sur les ondes au lieu des flashs d'information incessants ${ }^{(85)}$. Le 16,

75 Robert d'Harcourt, «La rue sanglante», C, 29.06.1953.

76 «En Allemagne orientale, mesures de clémence ... et autocritique», C, 29.06.1953.

77 «Dibrowa: Vorerst kein normaler Verkehr», FAZ, 01.07.1953.

78 «Differenzen im Ministerrat der Sowjetzone?», FAZ, 04-05.07.1953.

79 «Offene Gegensätze in Pankow», FR, 04-05.07.1953.

80 «Normalisierung in der Sowjetzone - nur äußerlich», SZ, 08.07.1953.

81 «Wachsender passiver Widerstand in den Betrieben der Sowjetzone», FR, 08.07.1953.

82 «'Neuer Kurs' in der Sowjetzone wird fortgesetzt», FR, 13.07.1953.

83 «Politische Hygiene», FAZ, 14.07.1953.

84 Karl-Eduard von Schnitzler était, en sa qualité de commentateur en chef de la radio Deutschlandsender, une célébrité parmi les journalistes est-allemands. Son renom s'accrut lorsqu'à partir de 1960, il anima à la télévision l'émission politique de propagande Der schwarze Kanal. 
Le Monde, fidèle au modus operandi des journaux français, fustige une fois de plus le régime, tirant «le plus péremptoire des arguments» des déclarations de M. Grotewohl, selon lesquelles jamais les provocateurs de Berlin-Ouest n'auraient pu «égarer» tant de travailleurs honnêtes, si «des fautes aussi lourdes qu'innombrables n'avaient pas été commises par le gouvernement de Pankow» ${ }^{(86)}$. Le correspondant concède néanmoins, à l'actif de l'Ouest, le rôle de la radio américaine, la RIAS, qui a contribué à diffuser dans tout le territoire les nouvelles des troubles de la capitale ${ }^{(87)}$.

Après ce tour d'horizon sur le régime, nous établissons le même constat de similarité entre journaux conservateurs et progressistes, toujours à l'exclusion de L'Humanité, et entre journaux allemands et français, ce qui confirme la thèse d'une pensée transnationale. Néanmoins, on observe une tendance plus nette de la presse française à dresser un réquisitoire impitoyable à l'égard du régime, vraisemblablement imputable à la présence d'un fort parti communiste en France et à la nécessité pour les libéraux de démontrer son inanité.

\section{Une réprobation de la répression partagée par tous les journaux}

Le 18 juin, toute la presse déplore les nombreux morts et blessés sous les tirs de la police et de l'Armée rouge. Le lendemain, tous les journaux signalent l'exécution du Berlinois de l'Ouest Willy Göttling, censé avoir agi pour le compte d'un service étranger de renseignements ${ }^{(88)}$. La FAZ compare Berlin-Est à une ville assiégée et fait un parallèle avec 1945 et l'entrée de l'Armée rouge dans la ville. Elle rapporte ensuite que 16 personnes ont été tuées par les troupes soviétiques et les policiers à BerlinEst ${ }^{(89)}$. La Süddeutsche Zeitung attire l'attention sur la pénurie de marchandises dans les magasins d'alimentation et l'interdiction donnée par les Soviétiques et la police aux habitants de Berlin-Ouest de rejoindre leur lieu de travail à l'Est ${ }^{(90)}$. Le Figaro recourt à l'anecdote teintée de couleur locale pour illustrer la rigueur du régime:

«J'ai essayé de m’approcher des Vopos [...] : “N'avancez pas!” me cria l'un d'eux. Je répondis: "Je suis française et journaliste." Mais lui, esquissant le geste de tirer son pistolet, hurla: "Pas un pas de plus, vous ne passerez pas" ${ }^{(91)}$.

Le 20-21 juin, Le Monde quant à lui se complaît, comme souvent, dans la mise en scène dramatique:

«Depuis la Potsdamer Platz, on peut apercevoir la perspective de la Leipzigerstrasse, la rue des ministères. [...] des deux côtés de la rue, on distingue la masse sombre des T-34 immobiles, rangés en ordre de bataille. Devant eux, face à l'Ouest, un cordon de soldats de la police populaire militarisée, en uniforme vert olive, la carabine à la main, interdit toute tentative de pénétrer en secteur oriental. L'audacieux qui tenterait d'en faire l'expérience serait fusillé sur place» ${ }^{(92)}$.

86 Roland Delcour, «Les agents occidentaux n'ont été pour rien dans le déclenchement de l'émeute populaire», $M, 16.07 .1953$

87 Ibid.

88 Par exemple, «16 Tote in Ost-Berlin», W, 19.06.1953.

89 «Das erste Todesurteil in Ost-Berlin vollstreckt», FAZ, 19.06.1953.

90 «Ostberlin vollkommen abgeriegelt», SZ, 19.06.1953.

91 D. AuClères, “'J'ai vu les troupes soviétiques reprendre en main la situation'» (note 52).

92 R. Delcour, "'L'ordre règne à Berlin-Est'» (note 27). Les T-34 sont des chars soviétiques. 
Le correspondant dépeint un climat de guerre:

«Derrière les soldats, entre les tanks, la rue est vide, jonchée de débris, de panneaux de propagande carbonisés, de pavés arrachés. Un silence de mort règne, que troublent par intermittence le claquement sec d'un coup de feu ou le tintamarre des chaînes d'un char " ${ }^{\left({ }^{3}\right)}$.

Le 22 juin, Die Welt annonce que les arrestations massives, que la Frankfurter Rundschau chiffre à plus de 10000 , doivent être stoppées sur ordre soviétique ${ }^{(94)}$, et mentionne la condamnation à mort d'Erna Dorn, cette ancienne commandante du camp de Ravensbrück libérée par les manifestants et accusée d'avoir pris la tête des protestations ${ }^{(95)}$. Le 23, Le Figaro, avec plus de détails que les autres journaux, relate qu'un jeune Berlinois de l'Ouest a été abattu dans le secteur britannique de Berlin par des tirs en provenance de l'Est après qu'il eut envoyé des cailloux sur des policiers. Pathos ici aussi: "Il y aura un mort de plus à déplorer, un enfant de plus qui, ce soir, ne rentrera pas à son foyer » ${ }^{(96)}$. Le 24 juin, la FAZ enregistre une augmentation du flux des réfugiés de l'Est et un nombre considérable (218) de Berlinois de l'Ouest portés disparus, renvoyant aux peines de pénitencier prononcées par des tribunaux est-allemands à l'encontre d'Ouest-Allemands ${ }^{(97)}$. Le Figaro cite le cas du maire communiste d'une petite ville fusillé par les Soviétiques pour avoir empêché des Vopos de tirer sur des manifestants ${ }^{(98)}$. On décèle ici encore la tendance de la presse française à insister particulièrement sur la nocivité du régime. Le 30 juin, Le Figaro évalue entre 30 et 68 le nombre des exécutions perpétrées dans la zone Est et croit savoir que 13700 des 40000 ouvriers arrêtés sont encore en prison ${ }^{(99)}$.

Le 2 juillet, Le Monde précise que si le couvre-feu est supprimé, l'état de siège et la loi martiale sont maintenus ${ }^{(100)}$. Le 3, Le Figaro, confirmant la propension de la presse française à une condamnation rigoureuse du régime, cite deux réfugiés de Berlin-Est échappés d'un abattoir où s'entassent 1300 prisonniers, dans des conditions inhumaines $^{(101)}$. Quant à L'Humanité, elle met au contraire l'accent sur le grand nombre de personnes libérées de prison ${ }^{(102)}$. Le 10, la Süddeutsche Zeitung évoque les banderoles rouges déployées à Berlin-Est avec la mention «Nous avons confiance dans notre gouvernement», alors que les uniformes et les mitraillettes dans les rues attestent à quel point le gouvernement se fie peu à la «confiance» de la population ${ }^{(103)}$. On décèle ici sous une plume ouest-allemande la propension plutôt française à condamner le régime. Le 11 juillet, la $F A Z$ chiffre à 12000 le nombre de personnes emprisonnées

93 Ibid.

94 «Verhaftungen gestoppt?» (note 56).

95 «Standgericht in Jena» (note 60).

96 Dominique Auclères, «Un nouvel incident hier, à Berlin - Un jeune homme est abattu par les 'vopos' en secteur britannique», $F, 23.06 .1953$.

97 «23 Standgerichtsurteile in der Sowjetzone», FAZ, 24.06.1953.

98 «Le maire communiste d'une ville d'Allemagne orientale aurait été fusillé par les Russes», F, 24.06.1953.

99 «Les communistes sont obligés de prendre de nouvelles mesures », F, 30.06.1953.

100 «Le couvre-feu est supprimé à Berlin-Est», $M, 02.07 .1953$.

101 «Treize cents Berlinois sont entassés à l'abattoir de Friedrichsfelde», F, 03.07.1953.

102 «Libération de 7700 détenus en République Démocratique Allemande», H, 04-05.07.1953.

103 «Aufgepflanzte Bajonette vor Ostberliner Ministerien», SZ, 10.07.1953. 
depuis le 17 juin, revoyant à la baisse le chiffre publié par Le Figaro $^{(104)}$. Le 13, le FAZ communique le rejet par le gouvernement de Grotewohl de l'aide alimentaire proposée par les Américains ${ }^{(105)}$, et, parmi d'autres journaux, annonce la levée de l'état d'exception par le haut commandement soviétique pour Berlin-Est ${ }^{(106)}$. L'Humanité s'insurge que les Américains proposent, "pour appuyer les mensonges de leur propagande sur la situation économique de l'est de l'Allemagne, d'y envoyer des vivres », point d'exclamation, "offre insolente et hypocrite» ${ }^{(107)}$.

Le 15, le quotidien décrit le véritable «pèlerinage» de Berlinois de l'Est en direction de Kreuzberg, situé à l'Ouest, où le maire d'arrondissement avait mis en place des centres de distribution alimentaire ${ }^{(108)}$. Selon le journal, la prochaine récolte est compromise par l'exode massif des paysans, le manque de moyens de transport ainsi que de pièces de rechange pour les machines agricoles et la collectivisation forcée ${ }^{(109)}$. Die Welt cite le ministre de la Sécurité Wilhelm Zaisser reconnaissant que 383 personnes ont trouvé la mort lors des troubles du 17 juin, auxquelles s'ajoutent 141 personnes exécutées et 14 condamnées à mort - en contradiction avec les 25 morts reconnus jusqu'alors par le régime ${ }^{(110)}$. On décèle ici la propension de tous les journaux, hormis L'Humanité, à s'appesantir sur les minimisations, voire les mensonges, des autorités est-allemandes.

Cette partie sur la répression permet elle aussi d'appuyer la thèse d'une pensée conservatrice et d'une pensée progressiste transnationales, de mettre en lumière la propension à la mise en scène dramatique du Monde et au pathos du Figaro et de pointer les durs verdicts de la presse française envers le gouvernement est-allemand.

\section{Le rôle des Soviétiques et la problématique de l'unité allemande - abordée plus volontiers par la presse ouest-allemande}

Le 17 juin, Le Figaro suggère que cette manifestation aurait pu être montée par les communistes pour prouver au monde la libéralité du régime ou bien fomentée par la Haute-commission soviétique pour se débarrasser des dirigeants est-allemands ${ }^{(111)}$. Le lendemain, la $F A Z$ conclut que les événements placent les autorités soviétiques devant la question du maintien en place ou non du gouvernement est-allemand ${ }^{(112)}$. La Frankfurter Rundschau discerne dans cette insurrection une vraie chance pour la question allemande, car elle contraint l'URSS à l'action ${ }^{(113)}$. Selon la Süddeutsche Zeitung, le gouvernement Grotewohl-Ulbricht n'est provisoirement plus en fonction, Berlin-Est ayant été placée sous la loi martiale des occupants. Le soulèvement serait imputable au changement de politique imposé par Moscou, qui a conduit les masses à aspirer à la

104 «12000 Personen seit dem 17. Juni verhaftet», FAZ, 11.07.1953.

105 «Amerikanische Hilfe für die Sowjetzone abgelehnt», FAZ, 13.07.1953.

106 «Der Ausnahmezustand in Ost-Berlin aufgehoben", FAZ, 13.07.1953.

107 "Après l'échec de leur coup de force à Berlin, les Américains offrent insolemment une 'aide' à l'Allemagne démocratique», $H, 13.07 .1953$.

108 «Nachbarhilfe über die Sektorengrenze», SZ, 15.07.1953.

109 «Ernte in der Sowjetzone gefährdet», SZ, 15.07.1953.

110 «Zaisser : 569 Tote bei den Juni-Unruhen», W, 15.07.1953.

111 «Des milliers d'ouvriers conspuent le gouvernement Grotewohl», F, 17.06.1953.

112 «Der Ausnahmezustand über Berlin verhängt» (note 11).

113 «Ulbricht und Grotewohl: Wir irrten» (note 49). 
disparition totale de leurs chaînes. Le journal indique que seuls les chars soviétiques ont pu empêcher une révolution de plus vaste ampleur ${ }^{(114)}$. Le 19 juin, la $F A Z$ évoque la remarque de Staline selon laquelle le communisme va aux Allemands «comme une selle à une vache » et le fait que l'Union soviétique a déjà envisagé d'abandonner la RDA afin de la marchander sur le plan diplomatique ${ }^{(115)}$. La Süddeutsche Zeitung expose des conjectures sur la réaction de Moscou: va-t-on y blâmer le haut-commissaire Vladimir Semionov, l'artisan du changement d'orientation, ou bien lui donner plein pouvoir pour engager la voie de la réunification ${ }^{(116)}$ ? Le Monde dément par ailleurs la thèse d'une action tolérée par les Soviétiques afin de justifier la liquidation du régime pour privilégier celle d'une «authentique explosion du mécontentement populaire " ${ }^{(117)}$. La Croix se déclare quant à elle convaincue que l'unité allemande, accompagnée d'une neutralisation, est l'objectif des Soviétiques - rencontrant en cela les vœux des manifestants ${ }^{(118)}$.

Le 23 juin, la $F A Z$ se déclare convaincue qu'une voie est désormais proscrite aux Soviétiques: la tentative «de conférer à la dictature une sorte de légitimité historique et révolutionnaire en prétendant qu'elle s'appuie sur un large mouvement populaire [...]. Les derniers vestiges de la fidélité à la tradition socialiste [...] sont maintenant anéantis " ${ }^{(119)}$. Le 24, selon La Croix, des indices autoriseraient à penser que les Soviétiques envisagent la destitution du gouvernement. Le journaliste estime qu'il aurait été facile à ceux-ci de jeter Ulbricht en pâture aux manifestants tout en maintenant Grotewohl - qui, issu de la social-démocratie, pouvait faire office de conciliateur -, mais que les huées de la foule contre le ministre-président ont déjoué leurs plans ${ }^{(120)}$. Les 4-5 juillet, la Frankfurter Rundschau n'exclut pas que Moscou se livre à un double jeu, d'encouragement des partis bourgeois et de soutien à Ulbricht ${ }^{(121)}$. Le 10 , la $F A Z$ enregistre la pression croissante exercée par les Soviétiques sur le gouvernement est-allemand pour qu'il mette rapidement des réformes en œuvre, afin d'offrir une base de négociations plus favorable avec les Occidentaux sur la question allemande ${ }^{(122)}$. En regard avec le rôle des Soviétiques, l'identité de vues entre la presse française et ouest-allemande prend un tour singulier: elle atteste que, si peu de temps après la guerre, les impératifs de la guerre froide l'emportent sur les ressentiments issus de l'antagonisme franco-allemand.

Une autre thématique, quant à elle propre à la presse ouest-allemande, se situe en lien étroit avec ce qui précède: l'appel à la solidarité des Allemands de l'Ouest avec leurs «frères» de l'Est, lancé notamment par la FAZ le 22 juin:

«Les habitants de l'Allemagne centrale [ont le droit] d'exiger de ceux, plus heureux, qui partagent leurs convictions dans un État libre, qu'ils se joignent à eux. [...] Lorsque la zone soviétique souffre de la faim, lorsqu'on lui impose des normes, lorsque les chars soviétiques s'avancent, tout cela se produit en vérité aussi contre et à l'Ouest » ${ }^{(123)}$.

114 «Ausnahmezustand in Ostberlin», SZ, 18.06.1953.

115 Paul Sethe, «Dürfen wir hoffen?», FAZ, 19.06.1953.

116 W. FriedmanN, «Signal für Ost und West» (note 19).

117 R. Delcour, «Les autorités de Berlin-Est ont perdu le bénéfice des mesures de détente» (note 53).

118 François Roussel, «Une explosion de colères depuis trop longtemps contenues », C, 19.06.1953.

119 «Entschleiert», FAZ, 23.06.1953.

120 «Les Soviétiques sacrifieront-ils le gouvernement Grotewohl et la SED? », C, 24.06.1953.

121 «Offene Gegensätze in Pankow» (note 79).

122 «Die Sowjets dringen auf weitere Erleichterungen», FAZ, 10.07.1953.

123 «Die Freiheit verdienen», FAZ, 22.06.1953. 
Le même quotidien indique le lendemain que la plus grande déception des Allemands de l'Est tient au fait que le soulèvement n'ait pas incité les Occidentaux à libérer la zone orientale $^{(124)}$ - où l'on reconnaît l'appel implicite à la solidarité. Deux jours plus tard, l'éditorialiste du journal sacrifie à l'emploi du pathos, dont il est peu coutumier: "Les 18 millions d'habitants sont animés par une haine profonde contre la tyrannie qui ne recule pas même devant le sacrifice d'une vie ${ }^{(125)}$. Le journaliste en tire l'injonction aux Allemands de l'Ouest de venir en aide aux opprimés et l'exhortation à envisager la réunification comme objectif vital, autre trait caractéristique de la presse ouest-allemande.

\section{Articles de fond prisés par la presse française et témoignages directs}

Nous concluons cet aperçu par un chapitre rapide sur une autre inclination de la presse française: dès le 18 juin, Le Monde propose aux lecteurs un article de fond avec portrait et biographie d'Ulbricht et un rappel circonstancié de l'histoire de la RDA ${ }^{(126)}$. Ce genre d'article n'est pas rare dans les journaux français, conscients du déficit de connaissances de leur public. Le 23, La Croix propose ainsi un long article dépassant l'actualité immédiate qui retrace la biographie des hommes forts de RDA, Ulbricht, Grotewohl et Wilhelm Zaisser, ministre de la Sécurité, et une présentation précise de l'histoire du pays, illustrant les efforts de la presse française pour informer sur le régime et la vie politique ${ }^{(127)}$. Le 25, Le Monde, témoignant une fois encore de cette volonté des quotidiens français, sollicite "un des meilleurs spécialistes de la question », Benno Sarel, pour proposer « une étude documentée sur la vie des ouvriers en zone soviétique, leurs conditions de travail, leur psychologie» ${ }^{(128)}$, où l'auteur affirme que le monde des usines qui a manifesté le 17 juin est le même que celui de la Semaine spartakiste de 1919. Le 6 juillet, Le Figaro, témoignant à son tour de cette détermination, fait un point détaillé sur la situation en RDA avant le soulèvement et livre un récapitulatif exhaustif des étapes de celui-ci sur tout le territoire. Non sans pathos, comme l'illustre cette évocation de la Friedrichstraße à Berlin: "C'est là que fut répandu le premier sang; c'est de là que partit la clameur de colère dont la zone entière se fit au centuple l'écho » ${ }^{(129)}$.

Une autre singularité, cette fois-ci partagée par plusieurs journaux toutes nationalités confondues, réside dans le goût pour le témoignage direct: le 23, la FAZ met en lumière le contraste qui règne à Berlin, entre la Berlinale à l'Ouest et l'émeute à l'Est ${ }^{(130)}$. L'article retranscrit ensuite le récit d'un témoin de Berlin-Est, qui illustre une peur panique des Russes et une atmosphère de siège: «Au moindre bruit de moteurs dans la rue, on se précipite à la fenêtre dans un demi-sommeil et on se surprend au réveil à

124 «Ein West-Berliner Junge an der Grenze erschossen », FAZ, 23.06.1953.

125 «In der ganzen Zone», FAZ, 25.06.1953.

126 Georges Penchener, "En retard d'un virage - Walter Ulbricht doit faire son mea culpa», $M$, 18.06.1953.

127 Pierre de Villemarest, «La disgrâce éventuelle du 'Lénine allemand' sera-t-elle la revanche du 'général Gomez' [Zaisser] ?...», C, 23.06.1953.

128 Benno SAREL, «Les émeutes ont été l'aboutissement d'un long effort de regroupement ouvrier», $M$, 25.06.1953.

129 Dominique Auclères, "Une zone de 18 millions d'hommes s'est révoltée contre Moscou», F, 06.07.1953.

130 Christian Ам Ende, «Angst vor den russischen Soldaten», FAZ, 23.06.1953. 
regarder de nouveau dehors » ${ }^{(131)}$. On distingue là un goût commun pour le témoignage direct et authentique, par l'intermédiaire d'un correspondant, d'un réfugié, ou d'un tiers qui fait part de ses impressions au journal. Le 10 juillet, le correspondant du Monde, racontant qu'il a parcouru le secteur Est de Berlin de long en large, où le calme ambiant contredit les rapports d'agence sur les troubles croissants, traduit le souhait marqué de la presse française de faire le point régulièrement, et ce, souvent, par le biais aussi d'un témoignage direct ${ }^{(132)}$.

À l'issue de cette étude, il apparaît donc qu'il n'existe pas sur le 17 juin 1953 de divergences d'interprétation entre les journaux progressistes et les quotidiens conservateurs, hormis le cas de L'Humanité, le seul à défendre la thèse propagée par les autorités est-allemandes de troubles fomentés par des agents occidentaux. On peut en déduire l'existence d'une pensée conservatrice et d'une pensée progressiste transnationales. Pas de différences d'interprétation non plus entre les publications ouest-allemandes et françaises, ce qui peut sembler surprenant si peu de temps après la guerre. La première conclusion est que la logique de la Guerre froide l'emporte sur l'ancien antagonisme franco-allemand et sur l'orientation politique des locuteurs. On n'a cependant pas affaire à une représentation uniforme: d'une part, les journaux n'optent pas pour les mêmes faits et n'en déduisent pas les mêmes effets. D’autre part, certains se révèlent plus sobres dans la description, comme la $F A Z$, et d'autres plus imagés et anecdotiques, comme la Süddeutsche Zeitung, ou sensationnalistes, comme Die Welt, voire prisent le pathos, comme Le Figaro ou Le Monde. Par ailleurs, la presse ouest-allemande lance fréquemment à ses lecteurs des appels à la solidarité avec leurs frères de l'Est et les exhorte à considérer l'unité allemande comme objectif essentiel. Les quotidiens français quant à eux livrent plus d'articles de fond sur la vie et le régime en RDA, conscients des lacunes de leur lectorat. Enfin, la presse française non communiste met l'accent sur l'aspect nocif du régime et s'efforce de démonter l'illusion d'un monde meilleur, ce qui peut tenir à la présence en France d'un puissant parti communiste dont il importe de saper l'influence. En tout état de cause, on peut conclure à l'existence d'un espace public (ouest)-européen durant les années 1950, dans lequel la représentation de la RDA fournit un apport à une vision transnationale des événements.

\section{Résumé}

La comparaison du traitement du 17 juin 1953 dans la Frankfurter Allgemeine Zeitung, la Süddeutsche Zeitung, la Frankfurter Rundschau et Die Welt d'une part, et Le Figaro, Le Monde, La Croix et L'Humanité d'autre part ne révèle aucune différence d'interprétation, que les journaux soient français ou allemands, ou bien conservateurs ou progressistes - hormis pour L'Humanité, qui adopte la thèse du gouvernement estallemand d'un putsch fomenté par des agents occidentaux. On décèle cependant des nuances de poids dans la représentation des événements: différences de ton entre des journaux plus portés sur le pathos, voire le sensationnalisme et d'autres plus réservés;

131 Ibid.

132 Roland Delcour, «Les chars russes n’ont pas quitté Berlin-Est», M, 10.07.1953. 
inclination de la presse allemande à appeler ses lecteurs à se solidariser avec les Allemands de l'Est et à garder à l'esprit l'objectif de l'unité allemande; goût de la presse française pour les articles de fond sur la RDA, à même de combler les lacunes de leur lectorat; et propension de cette même presse pour une condamnation du régime est-allemand plus rigoureuse que sa consour allemande, sans doute du fait de l'existence d'un puissant parti communiste en France.

\title{
Zusammenfassung
}

Der Vergleich zwischen den Darstellungen des 17. Juni 1953 in der Frankfurter Allgemeinen Zeitung, der Süddeutschen Zeitung, der Frankfurter Rundschau und Der Welt einerseits, und Le Figaro, Le Monde, La Croix und L'Humanité andererseits weist keine Deutungsunterschiede auf, ob die Zeitungen deutsch oder französisch, konservativ oder fortschrittlich sind - außer für L'Humanité, die die These der ostdeutschen Regierung von einem durch westliche Agenten angezettelten Putsches übernimmt. Allerdings sind wichtige Nuancen zu erkennen: Stilunterschiede zwischen zum Pathos bzw. zum Sensationalismus tendierenden und eher nüchternen Zeitungen; Neigung der deutschen Presse, ihre Leser zur Solidarität mit den Ostdeutschen und dazu, das Ziel der deutschen Einheit nicht zu vernachlässigen, aufzurufen; Vorliebe der französischen Presse für Hintergrundartikel über die DDR, die geeignet sind, dem Wissensmangel ihrer Leserschaft entgegenzuwirken; und Hang derselben Presse zu einer Verurteilung des ostdeutschen Regimes, die sich vermutlich aufgrund der Existenz einer mächtigen kommunistischen Partei in Frankreich als unerbittlicher als in der deutschen Presse erweist.

\begin{abstract}
The comparison in treatment in treatment from June 17, 1953 in the Frankfurter Allgemeine Zeitung, the Süddeutsche Zeitung, the Frankfurter Rundschau and Die Welt on the one hand, and Le Figaro, Le Monde, La Croix and L'Humanite on the other does not reveal any difference in interpretation whether the newspapers are French or German or conservative or progressive - except for the L'Humanité which adopts the East-German stand of a putsch plotted by agents of the West. One detects, however, strong nuances: difference in tone between newspapers more turned to pathos or even sensationalism and other newspapers more reserved; an inclination of the German press to call on its readers to demonstrate solidarity with the East-Germans and to keep to the forefront the goal of German unity, the preference of the French press for in depth articles on the GDR, capable of filling the knowledge gap of their readers and the tendency of this same press to condemn more rigorously the East-German regime, undoubtedly due to the existence of a powerful communist party in France.
\end{abstract}

